\title{
Persuasion with Limited Communication
}

\author{
SHADDIN DUGHMI, University of Southern California \\ DAVID KEMPE, University of Southern California \\ RUIXIN QIANG, University of Southern California
}

We examine information structure design, also called "persuasion" or "signaling," in the presence of a constraint on the amount of communication. We focus on the fundamental setting of bilateral trade, which in its simplest form involves a seller with a single item to price, a buyer whose value for the item is drawn from a common prior distribution over $n$ different possible values, and a take-it-or-leave-it-offer protocol. A mediator with access to the buyer's type may partially reveal such information to the seller in order to further some objective such as the social welfare or the seller's revenue. We study how a limit on the number of bits of communication affects this setting in two respects: (1) How much does this constraint reduce the optimal welfare or revenue? (2) What effect does constraining communication have on the computational complexity of the mediator's optimization problem?

In the setting of maximizing welfare under bilateral trade, we exhibit positive answers for both questions (1) and (2). Whereas the optimal unconstrained scheme may involve $n$ signals (and thus $\log (n)$ bits of communication), we show that $O\left(\log (n) \log \frac{1}{\epsilon}\right)$ signals suffice for a $1-\epsilon$ approximation to the optimal welfare, and this bound is tight. This largely justifies the design of algorithms for signaling subject to drastic limits on communication. As our main result, we exhibit an efficient algorithm for computing a $\frac{M-1}{M} \cdot(1-1 / e)$ approximation to the welfare-maximizing scheme with at most $M$ signals. This result hinges on an intricate submodularity argument which relies on the optimality of a greedy algorithm for solving a certain linear program. For the revenue objective, the surprising logarithmic bound on the number of signals does not carry over: we show that $\Omega(n)$ signals are needed for a constant factor approximation to the revenue of a fully informed seller. From a computational perspective, however, the problem gets easier: we show that a simple dynamic program computes the signaling scheme with $M$ signals maximizing the seller's revenue.

Observing that the signaling problem in bilateral trade is a special case of the fundamental Bayesian Persuasion model of Kamenica and Gentzkow, we also examine the question of communication-constrained signaling more generally. Specifically, in this model there is a sender (the mediator), a receiver (the seller) looking to take an action (setting the price), and a state of nature (the buyer's type) drawn from a common prior. The state of nature encodes both the receiver's utility and the sender's objective as a function of the receiver's action. Our results for bilateral trade with the revenue objective imply that limiting communication to $M$ signals can scale the sender's utility by a factor of $O\left(\frac{M}{n}\right)$ in general, where $n$ denotes the number of states of nature. We also show that our positive algorithmic results for bilateral trade do not extend to communication-constrained signaling in the Bayesian Persuasion model. Specifically, we show that it is NP-hard to approximate the optimal sender's utility to within any constant factor in the presence of communication constraints.

Additional Key Words and Phrases: Signaling; bayesian persuasion; bilateral trade; pricing

Work supported in part by NSF grant CCF-1423618.

Contact information: \{shaddin, dkempe, rqiang\}@usc.edu

Due to space constraints, most proofs are omitted from this version. A full version including proofs is available on arXiv.

Permission to make digital or hard copies of all or part of this work for personal or classroom use is granted without fee provided that copies are not made or distributed for profit or commercial advantage and that copies bear this notice and the full citation on the first page. Copyrights for components of this work owned by others than ACM must be honored. Abstracting with credit is permitted. To copy otherwise, or republish, to post on servers or to redistribute to lists, requires prior specific permission and/or a fee. Request permissions from permissions@acm.org.

EC'16, July 24-28, 2016, Maastricht, The Netherlands. C Copyright (C) 2016 ACM 978-1-4503-3936-0/16/07 ...\$15.00.

http://dx.doi.org/10.1145/2940716.2940781 


\section{INTRODUCTION}

Strategic interactions are often rife with uncertainty and information asymmetry. Auctions and markets on the Internet feature sellers with privileged information regarding their products, and buyers with private information regarding their willingness to pay. The information structures which govern these interactions play a key role in determining the equilibria of the resulting games. In Akerlof's market for lemons [Akerlof 1970], for instance, information asymmetries between the buyers and sellers of used cars - buyers cannot distinguish good cars from bad whereas sellers can - can lead to the collapse of the entire market. In other cases, information asymmetries can serve a useful purpose; for example, a seller of advertising impressions may conflate different impressions in order to prevent "cherry picking" by advertisers, increase competition, and as a result increase her ${ }^{1}$ revenue [Levin and Milgrom 2010]. It is for these reasons that information structure design, also known as signaling, is emerging as a new mechanism design for information. This new frontier, like traditional mechanism design, raises deep algorithmic and complexity-theoretic questions whose exploration has only recently begun (see, e.g., [Bro Miltersen and Sheffet 2012; Dughmi 2014; Dughmi et al. 2014; Dughmi and Xu 2016; Emek et al. 2012; Guo and Deligkas 2013]).

Perhaps one of the most fundamental economic interactions governed by the presence or absence of information is bilateral trade between two parties [Mas-Collel et al. 1995]. In (a simplified form of) bilateral trade, one side can choose whether to participate in the trade, and by doing so would generate a social surplus which is private information to him. The other side can propose to take a fixed amount of the social surplus. Two particularly natural instantiations of bilateral trade are the following:

(1) Trade of an item between a seller (who has no value for the item) and a buyer via a posted price. The seller's posted price is the amount of surplus she proposes to take, while the buyer's valuation for the item, drawn from a commonly known distribution, is the amount of social surplus the trade would generate. The buyer chooses whether to accept the seller's posted price. We call the resulting game the pricing game.

(2) Trade between an employer and an agent: ${ }^{2}$ an employer would like to hire an agent to complete a project, and has (known) utility $u$ for its completion. The agent has a private cost $c$ (drawn from a known distribution) for completing the project, and the social surplus generated is the difference $u-c$. Without knowing the cost of the agent, the employer posts a proposed payment $p$, which is equivalent to posting the share $u-p$ of the social welfare which she proposes to keep. We call this game the employment game.

The buyer/agent is assumed to be rational with quasilinear utility, while the seller/employer aims to maximize her own utility. The buyer/agent accepts an offer if he would derive non-negative utility from it, and rejects it otherwise; this corresponds to the valuation exceeding the price in the pricing game, and the payment exceeding the cost in the employment game. For concreteness, we will state all of our results in the language of the pricing game; however, all results carry over verbatim to the employment game, and we will occasionally remark on the interpretation of results in this context.

The seller's chosen offer price to the buyer depends on what she knows about the buyer's value. If the seller has no information other than the distribution $\Gamma$ of values in the population of potential buyers, she chooses the price $P^{*}=P^{*}(\Gamma)$ max-

\footnotetext{
${ }^{1}$ For clarity, we will throughout use female pronouns for the seller and male pronouns for the buyer.

${ }^{2}$ In the literature, this falls into the class of principal-agent models. However, in this article, we use the word "principal" for a different role, so we will use the non-standard nomenclature to avoid misunderstandings.
} 
imizing her revenue $\operatorname{Rev}(P, \Gamma)=P \cdot \operatorname{Pr}_{V \sim \Gamma}[V \geq P]$. This leads to a revenue of $\operatorname{Rev}(\Gamma)=\operatorname{Rev}\left(P^{*}(\Gamma), \Gamma\right)=P^{*} \cdot \operatorname{Pr}_{V \sim \Gamma}\left[V \geq P^{*}\right]$ for the seller, and a social welfare of Welfare $(\Gamma)=\operatorname{Pr}_{\Gamma}\left[V \geq P^{*}\right] \cdot \mathbb{E}_{V \sim \Gamma}\left[V \mid V \geq P^{*}\right]$ for both players combined. At the other extreme is the case when the seller is fully informed about $V$. She can now set a price $P=V$; trade will always occur, leading to a maximum social welfare of $\mathbb{E}_{V \sim \Gamma}[V]$, which is fully extracted as revenue by the seller. Notice the difference caused by different amounts of information being communicated: with no information, the social welfare can be arbitrarily smaller than with full information.

In order to fully inform the seller, very fine-grained information had to be communicated. In reality, for practical and logistical reasons, the information received by the seller about the buyer is typically limited. For example, in the employment game, the information may be provided by a university or certification agency, which may initially only be able to communicate a coarse-grained rating of the agent via a GPA or the performance on a certification test. When the "agent" provides a product (e.g., a piece of clothing or medication), the location or label it is sold under (boutique/brandname vs. discount/generic) sends a coarse signal to the potential employer/buyer about the distribution of qualities she is to expect. Indeed, the study of communication constraints and their impact on the outcomes of games dates back at least to the work of [Blumrosen and Feldman 2006; Blumrosen et al. 2007] on communication constraints in auctions.

How to optimally inform the seller with limited communication is the subject of the present article. ${ }^{3}$ This question actually comprises two separate thrusts: (1) What is the inherent price of limited communication? In other words, how much social welfare is lost because the principal can only communicate limited information to the seller? A particularly stark version of this question is the following: in the presence of a selfinterested seller, how much social welfare can a principal salvage by sending a single bit of information, which is equivalent to merely being able to exclude some buyers from the market? (2) What are the algorithmic consequences of limited communication? How well can the principal optimize the welfare with limited communication, if the computation has to be efficient as well?

In order to formalize the notion of limited communication, we first define information structures. At its most general, an information structure for the seller is a (possibly randomized) $\operatorname{map} \varphi$ from the realized value $V \in \mathbb{R}$ of the buyer to a signal $\sigma \in \Sigma$ presented to the seller. This in effect partitions the probability histogram of $\Gamma$ - or equivalently, the population of buyers - into different segments, with each corresponding to a signal. Specifically, we write $\varphi(v, \sigma)=\operatorname{Pr}[\varphi(v)=\sigma \mid V=v]$, where the randomness is over the internal coins of $\varphi$. Receiving signal $\sigma$ induces, via Bayes' rule, a posterior distribution $\mu_{\sigma}$ for the seller, characterized by $\operatorname{Pr}_{\mu_{\sigma}}[V=v]=\frac{\operatorname{Pr}_{\Gamma}[V=v] \cdot \varphi(v, \sigma)}{\operatorname{Pr}[\varphi(V)=\sigma]}$; here, the randomness in the denominator is over both $\Gamma$ and the internal coins of $\varphi$. Upon receiving $\sigma$, the seller's optimal price is $P^{*}\left(\mu_{\sigma}\right)$ maximizing $\operatorname{Rev}\left(P, \mu_{\sigma}\right)$. This price induces a revenue of $\operatorname{Rev}\left(\mu_{\sigma}\right)$ for the seller and a social welfare of Welfare $\left(\mu_{\sigma}\right)$. The expected revenue and social welfare over all draws of the buyer's value and randomness in the scheme $\varphi$ are then given by $\operatorname{Rev}(\varphi, \Gamma)=\sum_{\sigma \in \Sigma} \operatorname{Pr}[\varphi(V)=\sigma] \cdot \operatorname{Rev}\left(\mu_{\sigma}\right)$ and Welfare $(\varphi, \Gamma)=\sum_{\sigma \in \Sigma} \operatorname{Pr}[\varphi(V)=\sigma] \cdot \operatorname{Welfare}\left(\mu_{\sigma}\right)$, respectively.

We now revisit our motivating examples. Suppose that the distribution $\Gamma$ has support $\left\{v_{1}, v_{2}, \ldots, v_{n}\right\}$. Then, $V$ can be precisely communicated to the seller by choosing a signal set $\Sigma=\{1, \ldots, n\}$ and setting $\varphi\left(v_{i}, i\right)=1$ (and $\varphi\left(v_{i}, j\right)=0$ for $i \neq j$ ). By way of contrast, the signaling scheme communicating no information to the seller is

${ }^{3}$ We note that an alternate interpretation of this goal is in terms of market segmentation. Specifically, how would a market designer optimally partition the market into a limited number of segments? 
implemented with $\Sigma=\{1\}$ and $\varphi\left(v_{i}, 1\right)=1$ for all $i$. Notice that the latter uses much lower "communication complexity," as measured by $|\Sigma|$. Also notice that both these extreme information structures are inherently algorithmically efficient: given an explicit representation of $\Gamma$ and a value $V$, it is trivial to compute $\varphi(V)$. For the design of signaling schemes, we use the constraint that $|\Sigma|=M$, for a given bound $M$, as the "low communication complexity" constraint.

We would like to understand the impact of the communication complexity on the social welfare that can be (in principle) achieved, and to analyze the algorithmic question of computing optimal signaling schemes $\varphi$ with limited communication complexity. The "gold standard" for social welfare is $\mathbb{E}_{V \sim \Gamma}[V]$, which we will call the full-information welfare.

Formally, we are interested in the following questions: Given an explicit representation of the distribution $\Gamma$ and a bound $M$ on the number of available signals, (1) What fraction of the full-information welfare can be obtained by a signaling scheme with $M$ signals? (2) Are there (approximately) optimal and computationally efficient signaling schemes for maximizing social welfare?

\section{Our Results}

Our first main result (proved in Section 3) shows that signaling schemes even with an extremely limited number of signals are surprisingly powerful in extracting welfare.

THEOREM 1.1. For any distribution $\Gamma$ with support size $n$, there is a single segment of the market (i.e., a non-negative vector indexed by buyer types that is pointwise upperbounded by the prior over buyer types) with social welfare at least a $\Theta(1 / \log n)$ fraction of the full-information welfare $\mathbb{E}_{V \sim \Gamma}[V]$. The $\Theta(1 / \log n)$ bound is tight.

Notice that this result is quite surprising. There are value distributions (such as equal-revenue distributions) under which the presence of a self-interested seller results in only a fraction $1 / n$ of the full-information welfare being realized. The theorem states that merely by excluding some buyers, the principal can improve this bound to $\Theta(1 / \log n)$. Perhaps a "natural" conjecture would have been that using $M$ segments, at most a fraction $O(M / n)$ of welfare could be attained in the worst case. The theorem and the subsequent corollary show that this conjecture is false. However, as we will see shortly, it is in fact true for the objective of maximizing the seller's revenue.

Applying Theorem 1.1 repeatedly yields Corollary 1.2, which shows that with a relatively small number of signals, we can get arbitrarily close to the full-information welfare.

COROLlary 1.2. For any $\epsilon>0$ and any distribution $\Gamma$ with support size $n$, there is a signaling scheme with $O(\log n \log (1 / \epsilon))$ signals which obtains a $(1-\epsilon)$ fraction of the full-information welfare.

In terms of the number of bits of communication required, Corollary 1.2 implies that communicating $O(\log \log n+\log \log (1 / \epsilon))$ bits extracts a $(1-\epsilon)$ fraction of the social welfare that could be extracted using $\log n$ bits.

Corollary 1.2 also has the following algorithmic implication: exhaustively searching over all signaling schemes with $\Theta(\log n \cdot \log (1 / \epsilon))$ signals, one obtains a QPTAS (quasipolynomial time approximation scheme).

Obtaining a truly polynomial-time algorithm appears quite a bit more challenging, although - as discussed in Section 8 - we currently do not have any hardness results, even for exact optimization. Our main technical result is Theorem 1.3, which shows that one can obtain a constant factor approximation to the social welfare in polynomial time. 
THEOREM 1.3. For any $M>1$, there is a polynomial-time $\frac{M-1}{M} \cdot(1-1 /$ e) approximation algorithm for the problem of implementing a welfare-maximizing signaling scheme with at most $M$ signals, given an explicit representation of $\Gamma$.

The proof of this theorem is quite involved. At the heart of it is a proof that the social welfare achieved from a set of signals is submodular. More precisely, the proof focuses on the social welfare accrued from all but one signal (which we denote by $\perp$ and call the garbage signal - it is a signal of buyer types for which our solution will not be credited with any reward). Each non-garbage signal $\sigma$ induces an optimal equilibrium price $P^{*}\left(\mu_{\sigma}\right)$ chosen by the seller. We can think of the computation of $\varphi$ as first choosing the set $S=\left\{P_{1}, P_{2}, \ldots, P_{M-1}\right\}$ of equilibrium prices of the optimum solution, and then choosing an optimal signaling scheme inducing these particular prices. The key insight is that the optimum social welfare with price set $S$ (and one garbage signal) is a monotone and submodular function of $S$. This fact is proved as Theorem 5.1 in Section 5. The proof relies heavily on a characterization of the optimal signaling scheme inducing the set $S$ of prices. While it is easy to see that, given the target prices $S=\left\{P_{1}, P_{2}, \ldots, P_{M-1}\right\}$, this optimal signaling scheme can be computed using a linear program (see Section 2), we require a better characterization, and thereto show (as Theorem 4.3 in Section 4) that it is also the output of a greedy algorithm.

Optimizing Seller Revenue. The reader may have noticed our focus on social welfare. Almost equally frequently studied is the objective of maximizing seller revenue. It turns out that results for seller revenue are much more straightforward, both technically and in terms of their implications. First, the communication constraint can severely curtail the seller's revenue: when the buyer's value is drawn from an equalrevenue distribution supported on a geometric progression of length $n$, the revenuemaximizing signaling scheme with $M$ signals recovers only an $O(M / n)$ fraction of the full-information revenue. On the other hand, computing the optimal signaling scheme for revenue maximization is straightforward (see Section 6):

THEOREM 1.4. The optimal signaling scheme for maximizing seller revenue groups buyers into $M$ contiguous segments by valuations, i.e., if the same signal $\sigma$ is sent for buyers with valuations $v<v^{\prime}$, then $\sigma$ is also sent for all buyers with valuations $v^{\prime \prime} \in\left[v, v^{\prime}\right]$. As a result, there exists a polynomial-time dynamic programming algorithm which, given an explicit representation of $\Gamma$ and a bound $M$ on the number of signals, computes a signaling scheme maximizing the seller's expected revenue.

In the employment game, Theorem 1.4 confirms (for the purpose of employer utility maximization) the generally agreed-upon form of grading or performance evaluation, wherein the highest performers are grouped together in one category ('A'), followed by the next highest category ('B'), etc. Such signaling schemes are generally not optimal if the goal is to maximize social welfare, and indeed, the optimum signaling scheme for welfare maximization can be fairly complex. As an example, consider a buyer distribution supported on types $(1,2,3,4)$ with probabilities $(2 / 12,1 / 12,2 / 12,7 / 12)$, respectively. When $M=2$ signals are allowed, the optimal signaling scheme obtains full welfare by sending signals with posterior unnormalized probabilities of $(2 / 12,1 / 12,0,1 / 12)$ and $(0,0,2 / 12,6 / 12)$.

Bayesian Persuasion. While our main focus is on bilateral trade, the framework of communication-bounded signaling naturally applies to other games as well. A more general setting has been termed Bayesian Persuasion by Kamenica and Gentzkow [2011]: a sender observes a random variable capturing the "state of the world," and can send a signal to a receiver. The receiver, based on the received signal, chooses an action. The utility of both the sender and the receiver depend on the state of the world 
and the chosen action, and are not necessarily aligned. Thus, the sender's goal is to design the information structure such that the receiver will choose actions which are in expectation beneficial to him.

Notice that signaling in bilateral trade fits in this framework. In the pricing game, the seller is the receiver, the buyer's valuation is the state of the world, and the sender is a market designer with the goal of maximizing the seller's revenue or social welfare. In the employment game, the employer is the receiver, the employee's cost is the state of the world, and the sender is an educational institution or crowdsourcing website aiming to generate welfare for its participants.

Our results for revenue in the pricing game already imply that the price of limited communication is high in some persuasion games. While it may be natural to conjecture that the submodularity property carries over from bilateral trade to more general persuasion games, this is not the case: we establish a strong hardness result for maximizing the sender's utility in general. (A more formal version of this theorem and the proof are given in Section 7.)

THEOREM 1.5. For any constant $c>0$, it is NP-hard to construct a signaling scheme approximating the maximum expected sender utility to within a factor $c$, given an explicit representation of a Bayesian Persuasion game and a bound $M$ on the number of signals.

\section{Related Work}

Our focus is on the classical model of bilateral trade (see [Mas-Collel et al. 1995, Chapter 23]). Our choice of protocol, namely the take-it-or-leave-it offer, is arguably the simplest mechanism for bilateral trade, and in the case of the pricing game corresponds to the revenue-optimal mechanism by the classical result of Myerson [1981]. The study of the impact of auxiliary information on trade - also known as third degree price discrimination - has a long history, starting at least as early as [Pigou 1920]. We refer the reader to [Bergemann et al. 2015] for an in-depth look at this economic literature.

The work most directly related to ours is that of Bergemann et al. [2015], who examine the effects of information in the same pricing game. Their main result is a remarkable characterization of buyer and seller expected utilities that are attainable by varying the information structure of the seller, i.e., by segmenting the market and allowing the seller to price discriminate between segments. They characterize the space of realizable pairs $(r, u)$ for which there exists an information structure $\varphi$ such that the seller's expected revenue is $r$ and the buyer's expected utility is $u:(r, u)$ is realizable if and only if $r \geq \operatorname{Rev}(\Gamma)$ (i.e., the seller at least matches her "uninformed" revenue), $u \geq 0$, and $r+u \leq \mathbb{E}_{V \sim \Gamma}[V]$.

Implicit in [Bergemann et al. 2015] is a family of algorithms - parametrized by the distribution $\Gamma$ and a realizable pair of utilities $(r, u)$ - which implement a signaling scheme $\varphi$ realizing the pair of utilities $(r, u)$. When $\Gamma$ is an explicitly-described distribution with support size $n$, the signaling schemes implicit in [Bergemann et al. 2015] are efficient; their runtime is a low-order polynomial in $n .{ }^{4}$ However, the most interesting signaling schemes implied in [Bergemann et al. 2015] - in particular those with largest and smallest $u$ - use as many signals as the support size of the buyer distribution $\Gamma$. This realization motivates our examination of schemes with limited communication.

Roesler and Szentes [2016] also study the impact of information revelation on bilateral trade. In their model, the buyer can observe a signal of his value for the item,

\footnotetext{
${ }^{4} \mathrm{~A}$ particularly beautiful example of the schemes implicit in [Bergemann et al. 2015] is the greedy algorithm achieving $r=\operatorname{Rev}(\Gamma)$ and $u=\mathbb{E}_{V \sim \Gamma}[V]-\operatorname{Rev}(\Gamma)$.
} 
and will pay for it if the conditional expected value is weakly larger than the price. The seller will choose the optimal monopoly price according to the buyer's information structure.

The signaling problem in bilateral trade is a special case of Bayesian Persuasion, as formalized by Kamenica and Gentzkow [2011], generalizing an earlier model by Brocas and Carrillo [2007]. Instantiations, variants, and generalizations of the Bayesian Persuasion problem have seen a flurry of interest in recent years. For example, persuasion has been examined in the context of voting [Alonso and Camara 2014], security [Rabinovich et al. 2015; Xu et al. 2015], multi-armed bandits [Kremer et al. 2014; Mansour et al. 2015], medical research [Kolotilin 2015], and financial regulation [Gick and Pausch 2012; Goldstein and Leitner 2013]. Dughmi and Xu [2016] also consider persuasion algorithmically. However, they focus on Bayesian Persuasion without any communication constraint, but allowing exponentially (or infinitely) many states of nature in the number of actions.

More generally, Bayesian Persuasion is a special case of optimal information structure design in games. Recent work in computer science has examined this question algorithmically, mostly in the context of auctions [Bro Miltersen and Sheffet 2012; Dughmi et al. 2015, 2014; Emek et al. 2012; Guo and Deligkas 2013]. In all these works, the uncertainty (i.e., state of nature) concerns the item being sold, rather than the type of the buyer as in our model. Nevertheless, [Dughmi et al. 2015, 2014] are related to our work in that they also examine communication-limited signaling schemes. The work of Dughmi [2014] examines the complexity of signaling in abstract twoplayer normal form games, while the recent work of Cheng et al. [2015] presents an algorithmic framework for tackling a number of (unconstrained) signaling problems.

An analogy can be drawn between our work and some of the work on auction design subject to communication constraints. Blumrosen et al. [2007] study single-item auctions in which bidders can only communicate a limited number of bits to the auctioneer. They show that even severe bounds on communication only lead to mild losses in welfare and revenue. Moreover, they show that bidders simply report an interval in which their value for the item lies when faced with an optimal auction. Blumrosen and Feldman [2006] study communication-constrained mechanism design in singleparameter problems more generally, and examine necessary and sufficient conditions under which a communication-constrained mechanism matches or approximates the optimal (unconstrained) mechanism.

\section{PRELIMINARIES}

Throughout, we use the following conventions for notation. Vectors are denoted by bold face. When we write $\boldsymbol{x} \leq \boldsymbol{y}$ for vectors $\boldsymbol{x}, \boldsymbol{y}$, we mean that $x_{i} \leq y_{i}$ for all $i$. We will frequently want to reason about the sums of entries of a vector over a given set of indices. We then write $x_{I}=\sum_{i \in I} x_{i}$. We also apply this notation for elements of a matrix $X=\left(x_{i, j}\right)_{i, j}$, writing $x_{I, J}=\sum_{i \in I} \sum_{j \in J} x_{i, j}$. We will particularly use this notation when $I, J$ are (closed or half-open) intervals of integers.

\subsection{Signaling Schemes}

When constructing a signaling scheme, we assume that the distribution $\Gamma$ of buyer valuations is given explicitly as input. In particular, this means that it must have finite support of size $n$. We assume that it is given by the valuations $v_{1}<v_{2}<\ldots<v_{n}$, and their associated probabilities $p_{1}, p_{2}, \ldots, p_{n}$, satisfying $\sum_{i} p_{i}=1$. We write $\boldsymbol{v}$ and $\boldsymbol{p}$ for the vectors of all these values and probabilities, respectively.

In the introduction, for ease of exposition, we described a signaling scheme $\varphi$ in terms of its conditional probabilities $\varphi(v, \sigma)=\operatorname{Pr}[\varphi(v)=\sigma \mid V=v]$. For the re- 
mainder of this article, we use notation differing in two ways: (1) since all values are of the form $v_{i}$, we can index the buyer types by $i$ instead of $v$, and (2) it is much more convenient to use unnormalized probabilities instead of conditional probabilities: $x_{i, \sigma}=\varphi\left(v_{i}, \sigma\right) \cdot \operatorname{Pr}_{\Gamma}\left[V=v_{i}\right]$ is the probability that the buyer's valuation is $v_{i}$ and the signal $\sigma$ is sent. The signaling scheme is then fully described by the matrix $X \in[0,1]^{n \times M}=\left(x_{i, \sigma}\right)_{i \in\{1, \ldots, n\}, \sigma \in\{1, \ldots, M\}}$, satisfying that $\sum_{\sigma} x_{i, \sigma}=p_{i}$. From now on, we will therefore simply refer to the signaling scheme as $X$ instead of $\varphi$.

We sometimes describe a signal $\sigma$ in isolation by a nonnegative type-indexed vector $\mathbf{0} \leq \boldsymbol{x}_{\sigma}^{\prime} \leq \boldsymbol{p}$. We call such a vector a segment of the market $\boldsymbol{p}$. Thus, a signaling scheme can be thought of as a family of segments, one per signal, whose sum is the entire market $p$.

As discussed in the introduction, upon receiving the signal $\sigma$, the seller will choose a price $P(\sigma)$ maximizing $P \cdot \operatorname{Pr}_{V \sim \mu_{\sigma}}[V \geq P]$. This price will always be one of the possible buyer valuations $v_{i}$, as any other price could be raised slightly without losing any buyers. Furthermore, by merging signals with the same price into one signal, without loss of generality, there are no two signals for which the seller chooses the same price [Kamenica and Gentzkow 2011]. Hence, any signaling scheme $X$ induces indices $k_{1}, k_{2}, \ldots, k_{M}$ such that upon receiving signal $\sigma$, the seller chooses price $v_{k_{\sigma}}$. Without loss of generality, we can rearrange the signals so that $k_{1}>k_{2}>\ldots>k_{M}$.

For any signal $\sigma$, the expected welfare resulting from $\sigma$ under $X$ is called the served social welfare and defined as $w_{X}(\sigma)=\sum_{i \geq k_{\sigma}} v_{i} \cdot x_{i, \sigma}$; the social welfare is then $W(X)=$ $\sum_{\sigma} w_{X}(\sigma)$.

We call the singaling scheme $X$ optimal for welfare if $X$ maximizes $W(X)$. If $W(X) \geq$ $\alpha W\left(X^{\star}\right)$, where $X^{\star}$ is optimal for welfare, we call $X$ an $\alpha$-approximation signaling scheme for welfare.

\subsection{Welfare and Sanitized Welfare}

While our goal for most of this article is to maximize the social welfare, it turns out that a slightly modified objective function is significantly more amenable to analysis, both in terms of positive results for bilateral trade and the hardness result for more general persuasion. Specifically, there is a designated garbage signal $\perp$, and any welfare accrued when $\perp$ is sent is discounted: we thus define the sanitized welfare of $X$ to be $\widetilde{W}(X)=\sum_{\sigma \neq \perp} w_{X}(\sigma)$. By designating the signal $\sigma$ minimizing $w_{X}(\sigma)$ as the garbage signal, we observe:

Proposition 2.1. For all signaling schemes $X$, we have that $\frac{M-1}{M} \cdot W(X) \leq$ $\widetilde{W}(X) \leq W(X)$.

In particular, any signaling scheme $X$ maximizing sanitized welfare to within a factor $\alpha$ also maximizes social welfare to within a factor $\frac{M-1}{M} \cdot \alpha$. Since we will always focus on sanitized welfare in the context of welfare maximization, to avoid having to write $M-1$ for the number of signals everywhere, we will explicitly assume that our signaling schemes can use $M$ signals in addition to the garbage signal $\perp$.

\subsection{Sanitized Welfare Maximization Given Price Points}

Conceptually, the task of designing a good signaling scheme can be divided into two steps: (1) Choose the seller's price points $k_{1}>k_{2}>\cdots>k_{M}$ for non-garbage signals; (2) Design a signaling scheme $X$ maximizing $\widetilde{W}(X)$ such that the seller's best response to each signal $\sigma$ is in fact $k_{\sigma}$.

Given the chosen price point indices $k_{1}, \ldots, k_{M}$, the goal of finding the welfaremaximizing signaling scheme is characterized by the following linear program. 


$$
\begin{array}{lll}
\text { Maximize } & \sum_{\sigma=1}^{M} \sum_{i=k_{\sigma}}^{n} v_{i} x_{i, \sigma} & \\
\text { subject to } & \sum_{\sigma=1}^{M} x_{i, \sigma} \leq p_{i} & \text { for all } i \quad \text { (probability) } \\
& v_{k_{\sigma}} \cdot \sum_{i \geq k_{\sigma}} x_{i, \sigma} \geq v_{k} \cdot \sum_{i \geq k} x_{i, \sigma} & \text { for all } \sigma, k \quad \text { (revenue) } \\
& x_{i, \sigma} \geq 0 & \text { for all } i, \sigma .
\end{array}
$$

The probability constraints capture that we do indeed have a valid signaling scheme (with the garbage signal being assigned all residual probabilities $p_{i}-\sum_{\sigma=1}^{M} x_{i, \sigma}$ ). The revenue constraints capture that it is a best response for the seller to set the price point $k_{\sigma}$ when receiving the signal $\sigma$.

Notice that for step (2), the LP (1) actually achieves the optimal solution for given price points. The approximation is necessary because for step (1), choosing the optimal price points appears more difficult.

\subsection{Bayesian Persuasion}

Bayesian Persuasion [Kamenica and Gentzkow 2011] is a natural and strong generalization of signaling in bilateral trade. The game involves a sender and a receiver, and is characterized by the following: (1) a distribution over states of nature $\omega \in \Omega,(2)$ a set $A$ of actions the receiver can choose from, and (3) utility functions $u_{S}(\omega, a), u_{R}(\omega, a)$ mapping the state of nature and action chosen by the receiver to the utilities obtained by the two players.

To illustrate these abstract concepts, for the pricing game, the state of nature was the buyer's valuation, and the receiver's actions were prices $P$. In the present work, we assume that the distribution over states of nature is given explicitly by the vector $\boldsymbol{p}$ of probabilities $p_{\omega}$.

As with the pricing game, the sender can choose a signaling scheme $X=$ $\left(x_{\omega, \sigma}\right)_{\omega \in \Omega, \sigma \in \Sigma}$ with $M$ signals. Upon receiving the signal $\sigma$, the receiver will choose an action $a(\sigma) \in A$ maximizing $\mathbb{E}_{\omega \sim \mu_{\sigma}}\left[u_{R}(\omega, a)\right]$, breaking ties in favor of the sender; here, $\mu_{\sigma}$ denotes the posterior distribution over states of nature conditioned on receiving signal $\sigma$.

The sender's utility from sending the signal $\sigma$ is $u(\sigma)=\sum_{\omega} x_{\omega, \sigma} \cdot u_{S}(\omega, a(\sigma))$, and he would like to maximize his overall expected utility $U(X)=\sum_{\sigma} u(\sigma)$. As with the special case of social welfare, we will mostly focus on the sanitized sender utility $\widetilde{U}(X)=\sum_{\sigma \neq \perp} u(\sigma)$ of signaling schemes with a dedicated garbage signal $\perp$; by the same argument as for social welfare, the sanitized sender utility is within at least a factor $\frac{M-1}{M}$ of the sender utility.

\section{WELFARE WITH LIMITED COMMUNICATION}

In this section, we provide a proof of Theorem 1.1. We then discuss some of its implications, including Corollary 1.2 and a QPTAS for the problem of maximizing social welfare subject to limited communication.

We begin by showing a special case of the theorem when each $v_{i}$ is a power of 2 . We then reduce the general case to this special case at a loss of a constant factor.

LEMMA 3.1. If each $v_{i}$ is a power of 2 , then the ratio of the full-information welfare to the maximum served social welfare of a single segment of the market is at most $O(\log n)$.

Proof. The lemma is trivial for $n=1$, so assume that $n \geq 2$. Let $\mathrm{SW}=\sum_{i} p_{i} v_{i}$ be the full-information welfare. First, we remove (i.e., exclude from the segment we 
construct) all types $i$ with $p_{i} v_{i}<\frac{\mathrm{SW}}{n^{2}}$. Because there are at most $n$ such types, this can decrease the full-information welfare by at most a factor of $1-1 / n$.

Now, we group all (remaining) types $i$ into $O(\log n)$ bins according to $p_{i} v_{i}$. Specifically, bin $B_{j}, j \geq 0$ contains all types $i$ with $p_{i} v_{i} \in\left(\frac{\mathrm{SW}}{2^{j+1}}, \frac{\mathrm{SW}}{2^{j}}\right]$. Since $p_{i} v_{i} \geq \frac{\mathrm{SW}}{n^{2}}$, there are at most $2 \log n$ bins. Thus, there is at least one bin $B_{j}$ such that $\sum_{i \in B_{j}} p_{i} v_{i} \geq \frac{n-1}{n} \cdot \frac{1}{2 \log n}$. SW. Fix such a $j$ for the rest of the proof, and define $u=\frac{\mathrm{SW}}{2^{j+1}}$.

Let $i^{*}=\min B_{j}$ be the type in $B_{j}$ corresponding to the smallest value. We define a market segment $\boldsymbol{q}$ as follows: Let $q_{i^{*}}=\frac{u}{v_{i^{*}}}$, let $q_{i}=\frac{u}{2 v_{i}}$ for all $i \in B_{j}$ with $i \neq i^{*}$, and let $q_{i}=0$ for $i \notin B_{j}$.

By definition of the bins, for all $i \in B_{j}$ we have $u<p_{i} v_{i} \leq 2 u$, and therefore $p_{i} / 4 \leq$ $q_{i} \leq p_{i}$. In particular, $\mathbf{0} \leq \boldsymbol{q} \leq \boldsymbol{p}$, and therefore $\boldsymbol{q}$ describes a valid segment of $\boldsymbol{p}$.

We next show that, when facing the market segment $q$, the seller will choose the price point $i^{*}$. The seller's revenue from choosing $i^{*}$ is at least $q_{i^{*}} v_{i^{*}}=u$. On the other hand, for any $i>i^{*}$, the seller obtains revenue at most $v_{i} \cdot \sum_{k \geq i, k \in B_{j}} \frac{u}{2 v_{k}} \leq v_{i} \cdot \frac{u}{2 v_{i}}$. $\sum_{k=0}^{\infty} \frac{1}{2^{k}}=u$; here, we used that the valuations are powers of 2 . Therefore, $v_{i^{*}}$ is the revenue-maximizing price for $q$. With the seller choosing $v_{i^{*}}$ as the price, the served social welfare of $q$ is

$$
\sum_{i \in B_{j}} q_{i} \cdot v_{i} \geq \frac{1}{4} \sum_{i \in B_{j}} p_{i} \cdot v_{i} \geq \frac{(n-1)}{8 n \log n} \cdot \mathrm{SW} \geq \frac{1}{16 n \log n} \cdot \mathrm{SW} .
$$

Thus, $\boldsymbol{q}$ is a segment with served social welfare at least $\frac{1}{O(\log n)} \cdot \mathrm{SW}$.

We utilize Lemma 3.1 to prove the upper-bound portion of Theorem 1.1. The idea is to round down all valuations to the nearest power of 2 (losing at most a factor of 2 in the welfare), then utilize Lemma 3.1 to construct a segment for the new valuation distribution achieving at least a factor $\Omega(1 / \log n)$ of the full-information welfare, and finally reconstruct a segment/signal for the original distribution. For the matching lower bound, we use an "equal welfare distribution."

Proof of Theorem 1.1. Let $v_{j}^{\prime}, 1 \leq j \leq m$, be the valuations of the original distribution, with associated probabilities $p_{j}^{\prime}$. The new valuations are $v_{i}=2^{i}$, with $p_{i}=\sum_{2^{i} \leq v_{j}^{\prime}<2^{i+1}} p_{j}^{\prime}$. We allow the index $i$ to be negative for notational convenience. Let $n$ denote the support size of $\boldsymbol{p}$, and note that $n \leq m$. Because valuations were reduced by at most a factor of 2 , the full-information welfare of the new distribution is at least half that of the original one, i.e., $\sum_{i} p_{i} v_{i} \geq \frac{1}{2} \sum_{j} p_{j}^{\prime} v_{j}^{\prime}$.

Let $\boldsymbol{q}$ be a single segment with served social welfare at least a $1 / O(\log n)$ fraction of the full-information welfare of $\boldsymbol{p}$. By Lemma 3.1, such a $\boldsymbol{q}$ exists. Let $i^{*}$ be the price point chosen by the seller under $\boldsymbol{q}$, and $R$ the seller's revenue. By optimality of $i^{*}$ for the seller, we get that

$$
R=v_{i^{*}} \cdot\left(q_{i^{*}}+\sum_{k=i^{*}+1}^{n} q_{k}\right) \geq v_{i^{*}+1} \cdot \sum_{k=i^{*}+1}^{n} q_{k}=2 v_{i^{*}} \cdot \sum_{k=i^{*}+1}^{n} q_{k} .
$$

Hence, $q_{i^{*}} \geq \sum_{k=i^{*}+1}^{n} q_{k}$, meaning that at least half of the probability mass, and thus half of the seller's revenue $R$ from $\boldsymbol{q}$, comes from type $i^{*}$. Now define a segment $\boldsymbol{q}^{\prime}$ of the original type distribution $\boldsymbol{p}^{\prime}$ as follows. For type $i^{*}$, take a total of probability mass $q_{i^{*}}$ from types $j$ with $2^{i^{*}} \leq v_{j}^{\prime}<2^{i^{*}+1}$. For all types $i>i^{*}$, take a total of probability mass $q_{i} / 8$ from types $j$ with $2^{i} \leq v_{j}^{\prime}<2^{i+1}$, and for types $j$ with $v_{j}^{\prime}<2^{i^{*}}$, set $q_{j}^{\prime}=0$. 
Observe that the full-information welfare of $\boldsymbol{q}^{\prime}$ is at least a $1 / 8$ fraction of the served social welfare of $q$. It remains to show that a constant fraction of that social welfare is above the seller's revenue-maximizing offer price for $\boldsymbol{q}^{\prime}$, which we do next.

First, note that the price $2^{i^{*}}$ gives the seller a revenue of at least $R / 2$, even just from all buyers of types $j$ with $2^{i^{*}} \leq v_{j}^{\prime}<2^{i^{*}+1}$. Next, consider a price of $v_{j}^{\prime} \geq 2^{i^{*}+1}$, say $2^{i} \leq v_{j}^{\prime} \leq 2^{i+1}$ with $i \geq i^{*}+1$. The revenue of such a price is at most

$$
v_{j}^{\prime} \cdot \sum_{k \geq j} q_{k}^{\prime} \leq 2^{i+1} \cdot \sum_{k \geq i} q_{k} / 8=\frac{1}{4} v_{i} \cdot \sum_{k \geq i} q_{k} \leq R / 4 .
$$

Hence, no price $v_{j}^{\prime} \geq 2^{i^{*}+1}$ can be revenue-maximizing for $\boldsymbol{q}^{\prime}$, and all types in the segment $\boldsymbol{q}^{\prime}$ with value at least $2^{i^{*}+1}$ are served. It remains to show that a constant factor of the remaining social welfare - associated with values between $2^{i^{*}}$ and $2^{i^{*}+1}$ - is also served.

Because the price $2^{i^{*}}$ dominates all prices $v_{j}^{\prime} \geq 2^{i^{*}+1}$, the seller will choose some price $j$ with $2^{i^{*}} \leq v_{j}^{\prime}<2^{i^{*}+1}$, and the chosen price must give the seller revenue at least $R / 2$. The calculation in (3) implies that the revenue extracted from types $j$ with $v_{j}^{\prime} \geq 2^{i^{*}+1}$ is at most $R / 4$. Hence, at least a revenue (and thus also social welfare) of $R / 4$ must come from types $j$ with $2^{i^{*}} \leq v_{j}^{\prime}<2^{i^{*}+1}$. By construction, the full-information welfare associated with those types is at most $2^{i^{*}+1} q_{i^{*}}=2 v_{i^{*}} q_{i^{*}}$, which is at most $2 R$ by (2). Consequently, at least a $1 / 8$ fraction of the total social welfare associated with those types is served, as needed.

In summary, $\boldsymbol{q}^{\prime}$ serves a constant fraction of the full-information welfare of $\boldsymbol{q}$, which in turn is a $1 / O(\log n) \geq 1 / O(\log m)$ fraction of the full-information welfare of the distribution $\boldsymbol{p}^{\prime}$. Hence, for any distribution supported on $m$ types, a single segment is enough to serve a $1 / O(\log m)$ fraction of the social welfare.

To show that this bound is tight, we construct an "equal-welfare distribution" with the property that no single segment extracts more than an $O(1 / \log n)$ fraction of the full-information social welfare. Let $v_{i}=2^{i}$ for $0 \leq i \leq n$, and $p_{i}=\frac{1}{2^{i} \cdot(n-i)}$ for $0 \leq i<n$, with $p_{n}=\frac{1}{2^{n-1}}=p_{n-1}$. Notice that these "probabilities" do not sum to 1 ; we omit the normalization constants for legibility, since they will cancel out in the subsequent calculations.

The full-information welfare of $\boldsymbol{p}$ is $\sum_{i=0}^{n} p_{i} v_{i}=2+\sum_{i=1}^{n} \frac{1}{i}=\Theta(\log n)$. Now consider any segment $\boldsymbol{q}$ of $\boldsymbol{p}$, and let $v_{k}=2^{k}$ be the seller's revenue-maximizing price for $\boldsymbol{q}$. If $k=n$, the served social welfare is $v_{n} q_{n} \leq v_{n} p_{n}=2=O(1)$, as needed. Assume now that $k<n$. For each $i$ with $k<i \leq n$, the optimality of the price $v_{k}$ implies that $v_{i} q_{[i, n]} \leq v_{k} q_{[k, n]}$, or equivalently $q_{[i, n]} \leq \frac{v_{k}}{v_{i}} q_{[k, n]}$. By choosing $i=k+1$, we get that $q_{[k+1, n]} \leq \frac{v_{k}}{v_{k+1}} q_{[k, n]}=\frac{q_{[k, n]}}{2}$, and consequently $q_{[k, n]} \leq 2 q_{k}$. We can now bound the served social welfare of $\boldsymbol{q}$ by a constant:

$$
\begin{aligned}
\sum_{i=k}^{n} v_{i} q_{i} & =v_{k} q_{[k, n]}+\sum_{i=k+1}^{n}\left(v_{i}-v_{i-1}\right) q_{[i, n]} \leq 2 v_{k} q_{k}+2 \sum_{i=k+1}^{n}\left(v_{i}-v_{i-1}\right) \frac{v_{k}}{v_{i}} q_{k} \\
& =2 v_{k} q_{k}\left(1+\sum_{i=k+1}^{n} \frac{v_{i}-v_{i-1}}{v_{i}}\right)=v_{k} q_{k}(n+2-k) .
\end{aligned}
$$

Because $q_{k} \leq p_{k}$, we can bound $v_{k} q_{k}(n+2-k) \leq v_{k} p_{k}(n+2-k)=\frac{n+2-k}{n-k} \leq 3$. 
In summary, any single segment can obtain at most constant welfare, and thus at most an $O(1 / \log n)$ fraction of the total social welfare for this instance.

To derive Corollary 1.2, simply pick segments greedily, always choosing the next segment to have largest possible served social welfare. By Theorem 1.1, each subsequent segment obtains at least a $\frac{1}{c \log n}$ fraction of the residual welfare at that point, meaning that after adding $c \log n \log (1 / \epsilon)$ signals, the fraction of the total welfare obtained by the signaling scheme is at least $1-\left(1-\frac{1}{c \log n}\right)^{c \log n \log (1 / \epsilon)} \geq 1-\epsilon$.

Remark 3.2. One can obtain a positive result very directly when the ratio $\rho=$ $\frac{\max _{i} v_{i}}{\min v_{i}}$ is bounded. Group all buyers according to their values into $\log \rho$ bins: for $u=\min _{i} v_{i}$, buyers with $2^{j} u \leq v_{i}<2^{j+1} u$ are put into bin $j$, By considering each bin as a segment, we can see that the revenue (also, the served social welfare) of each segment is at least half of the its full-information welfare. Choosing the best $M$ bins leads to a bound of $\Omega(\min (1, M / \log \rho))$. However, our result is of more interest when the ratio between the largest and smallest valuations can be very large.

To get a quasi-polynomial time approximation scheme (QPTAS) for the problem of finding a welfare-maximizing signaling scheme with at most $M$ signals, consider a desired approximation parameter $\epsilon$. We distinguish two cases. If $M \leq c \log n \log (1 / \epsilon)$ (again, $c$ is the constant in $O(\log n)$ in Theorem 1.1), enumerate all possible choices of price points, and find the truly optimal one (i.e., there is no approximation factor lost in this case). Notice that there are at most $\left(\begin{array}{c}n \\ M\end{array}\right)=O\left(n^{M}\right)$ such combinations to consider, and for each of them, either the LP (1) or the greedy algorithm (Theorem 4.3) allows us to evaluate the maximum welfare attainable with that set of prices. Thus, the running time is quasi-polynomial for fixed $\epsilon$.

When $M>c \log n \log (1 / \epsilon)$, by Corollary 1.2, choosing the $M$ price points greedily obtains a $(1-\epsilon)$ fraction of the full-information welfare. Thus, it certainly obtains the same fraction of the maximum that could be achieved with $M$ signals.

\section{SANITIZED WELFARE MAXIMIZATION WITH A GREEDY ALGORITHM}

In this section, we show that, given the set of price points, an optimal solution to the LP (1) (i.e., the problem of maximizing sanitized welfare) can be computed by a greedy algorithm constructing the signals one by one. Besides the faster running time, the main value of the greedy algorithm is as an analysis tool for the problem of choosing the (near-)optimal price points; the analysis for that problem is carried out in Section 5.

We begin with an algorithm for constructing just one signal $\sigma$ with a given price point $k=k_{\sigma}$. Let $\boldsymbol{p}^{\prime}$ be a vector of residual probabilities for buyer types, i.e., the probability of each type that has not been allocated to any signals previously. Hence, $0 \leq p^{\prime} \leq p$. The goal is to construct the probabilities $y$ for a single signal with price point $k$; that is, we require that $0 \leq y_{i} \leq p_{i}^{\prime}$ for all $i$, and the revenue constraint $v_{k} \cdot \sum_{j \geq k} y_{j} \geq v_{i} \cdot \sum_{j \geq i} y_{j}$ must be satisfied for all $i$. The revenue constraint can be written as

$$
y_{[i, n]} \leq \frac{v_{k}}{v_{i}} \cdot y_{[k, n]} \quad \text { for all } i .
$$

In an optimal (single) signal, for each $i \geq k$, either Inequality (4) must be tight, or $y_{i}=p_{i}^{\prime}$; otherwise, $y_{i}$ could be increased, raising social welfare. For the same reason, $y_{k}=p_{k}^{\prime}$. Because no buyer of type $i<k$ will ever purchase at price $v_{k}$, such buyers contribute 0 to revenue and (social or buyer) welfare. Hence, without loss of generality, the optimum signal has $y_{i}=0$ for all $i<k$.

These observations suggest the following algorithm: Gradually raise $y_{k}$ from 0 to $p_{k}^{\prime}$. Simultaneously raise the $y_{i}$ for $i>k$ in such a way that at all times, for each $i$, either 
$y_{i}=p_{i}^{\prime}$ or Inequality (4) is tight. This is accomplished by raising the tail sums $y_{[i, n]}$ at a rate of $\frac{v_{k}}{v_{i}}$ while $y_{[k, n]}$ is raised at rate 1 . Solving for the necessary rates of increase of individual $y_{i}$ gives rise to the algorithm CONSTRUCT-ONE-SIGNAL.

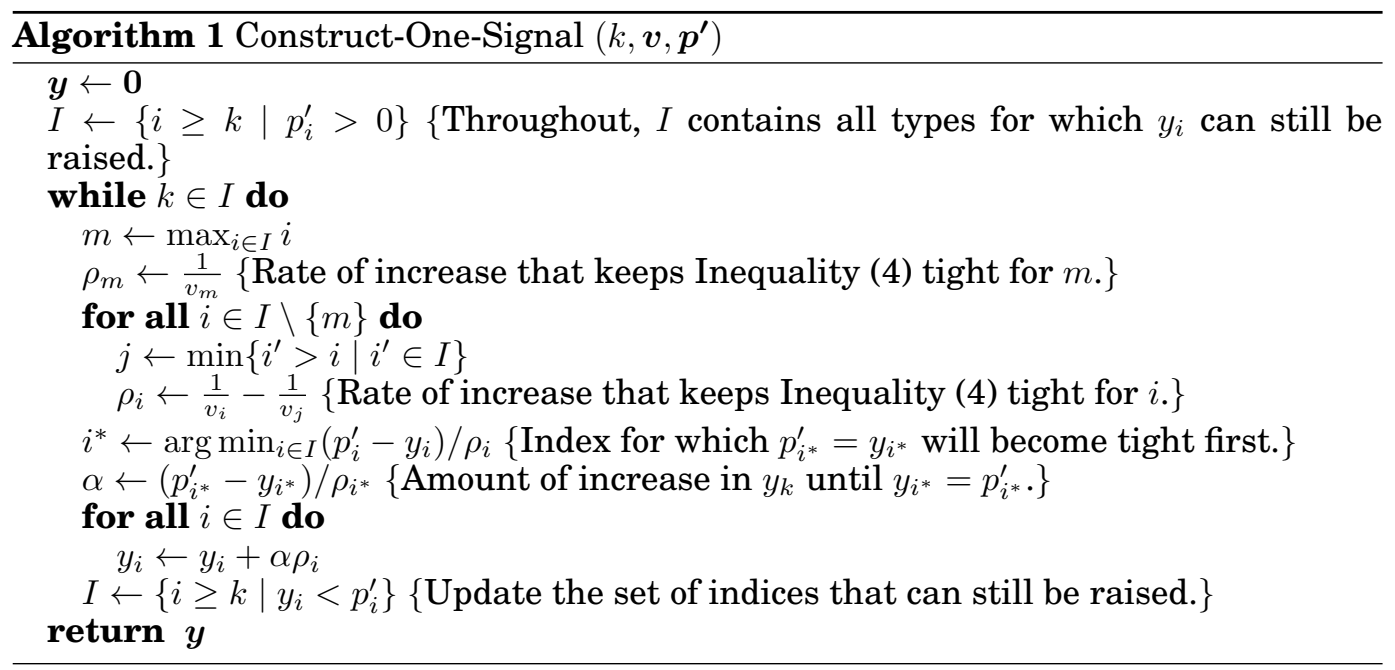

Our first lemma simply restates the revenue constraint, and captures the fact that if $y_{i}<p_{i}^{\prime}$ at any time during the algorithm, then the revenue constraint (4) must be tight for $i$. The proof is straightforward by induction on iterations.

LEMMA 4.1. For all indices $i$ and every step of the algorithm, $y_{[i, n]} \leq \frac{v_{k} \cdot y_{[k, i]}}{v_{i}-v_{k}}$.

Furthermore, if $i \in I$ at some point of the algorithm (including at termination), then at that point in time, $v_{i} \cdot y_{[i, n]}=v_{k} \cdot y_{[k, n]}$. The latter condition is equivalent to saying that $y_{[i, n]}=\frac{v_{k} y_{[k, i)}}{v_{i}-v_{k}}$.

The key property of the algorithm CONSTRUCT-ONE-SIGNAL is that it maximizes all tail sums $y_{[i, n]}$ :

LEMMA 4.2. Let $\boldsymbol{y}^{\prime} \leq \boldsymbol{p}^{\prime}$ be any signal. If $k$ is the chosen price by the seller under $\boldsymbol{y}^{\prime}$, i.e., $v_{k} \cdot y_{[k, n]}^{\prime} \geq v_{i} \cdot y_{[i, n]}^{\prime}$ for all $i$, then $y_{[i, n]} \geq y_{[i, n]}^{\prime}$ for all $i \geq k$.

Proof. For contradiction, assume that $y_{[i, n]}<y_{[i, n]}^{\prime}$ for some $i$, and fix the minimum such $i$. Let $j \geq i$ be the minimum index with $y_{j}<p_{j}^{\prime}$; note that such an index must exist, as otherwise, $y_{[i, n]}=\sum_{j \geq i} y_{j}=\sum_{j \geq i} p_{j}^{\prime} \geq \sum_{j \geq i} y_{j}^{\prime}=y_{[i, n]}^{\prime}$. As a result, the index $j \in I$ at the termination of the algorithm, implying by Lemma 4.1 that $v_{k} \cdot y_{[k, n]}=v_{j} \cdot y_{[j, n]}$. Now, we obtain the following contradiction:

$$
y_{[i, n]}=y_{[j, n]}+\sum_{i^{\prime}=i}^{j-1} y_{i^{\prime}}=\frac{v_{k} \cdot y_{[k, n]}}{v_{j}}+\sum_{i^{\prime}=i}^{j-1} p_{i^{\prime}}^{\prime} \geq y_{[j, n]}^{\prime}+\sum_{i^{\prime}=i}^{j-1} y_{i^{\prime}}^{\prime}=y_{[i, n]}^{\prime} .
$$

To construct a complete signaling scheme, we invoke the algorithm CONSTRUCTONE-SIGNAL repeatedly, constructing the signals one at a time. The important part here is that the signals must be constructed in decreasing order of the target price. The intuitive reason is that the inclusion of high-value buyers in a signal makes a higher price more attractive to the seller, thus posing additional constraints on the required probability mass of lower-valued buyers that must be included. Thus, it is 
always better to include as many high-valued buyers in the high-priced signals as possible, and this is accomplished by constructing those signals first. Hence, we assume that the signals are sorted in descending order of their prices.

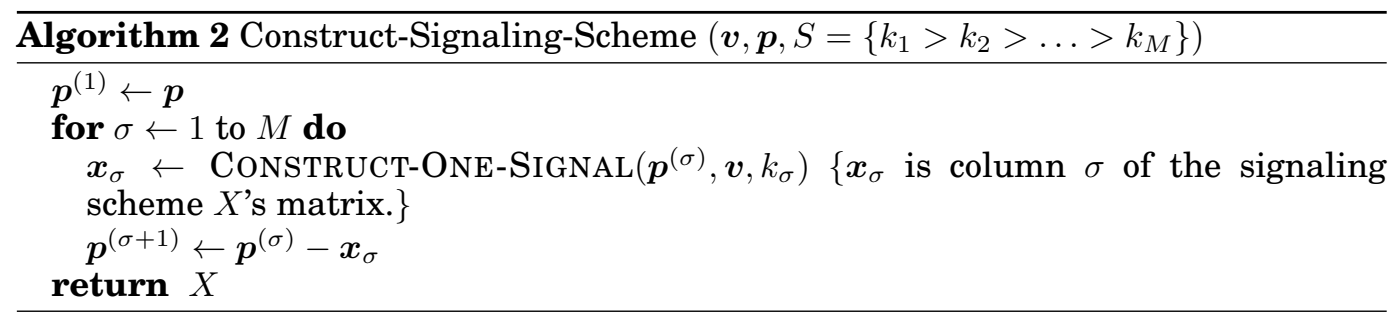

THEOREM 4.3. The algorithm CONSTRUCT-SignAling-SCHEME solves the linear program (1) optimally.

The proof of this theorem proceeds by showing that an optimal solution $X^{*}$ for the linear program (1) can be (gradually) transformed into the solution $X$ constructed by the algorithm CONSTRUCT-SIGNALING-SCHEME without decreasing its solution quality. It is technically fairly involved, and given in the full version.

\section{SUBMODULARITY OF SANITIZED WELFARE}

We prove that the sanitized welfare objective function $\widetilde{W}(S)$ is a submodular function of the set $S$ of chosen price points. (There is also a garbage signal $\perp \notin S$.) Let $S=\left\{k_{1}>\right.$ $\left.k_{2}>\ldots>k_{m}\right\}$ be $k$ price points. $\widetilde{W}(S)$ is defined as the optimum solution to the LP (1) with the given price points. We show the following:

THEOREM 5.1. If $S \subseteq T$ and $k \notin T$, then $\widetilde{W}(T \cup\{k\})-\widetilde{W}(T) \leq \widetilde{W}(S \cup\{k\})-\widetilde{W}(S)$.

Theorem 4.3 states that the greedy algorithm solves the problem for $S \cup\{k\}$ optimally, but the effects of adding $k$ are subtle. It is fairly easy to analyze what happens in the iteration when $k$ itself is added: that the welfare increase is larger for $S$ than for $T$ is easily seen by a simple monotonicity argument, presented in the full version. However, the addition of $k$ has "downstream" effects. The construction of subsequent signals with price points $k_{\sigma}<k$ will now face different residual probabilities, and the resulting reductions in those signals need to be carefully balanced against the gains from the signal with price point $k$.

Part of the complexity arises from the rather complex construction of the signal for price point $k$ itself. It is captured by the algorithm CONSTRUCT-ONE-SIGNAL, which itself runs through iterations in which different sets $I$ of indices have their probabilities increased. In order to eliminate this source of complexity, we will think of adding the signal with price point $k$ "gradually." Specifically, we consider the execution of CONSTRUCT-SIGNALING-SCHEME in which the execution of CONSTRUCT-ONESIGNAL for the signal with price point $k$ may be terminated prematurely. An upper bound $B$ on the tail probability is specified, and CONSTRUCT-ONE-SIGNAL is stopped when $\sum_{i \geq k} y_{i}=B$. After signal $k$ is constructed in this modified way, subsequent signals will be constructed normally by CONSTRUCT-SIGNALING-SCHEME.

A modification of the proof of Theorem 4.3 shows that this modified algorithm optimally solves the LP (1) with the added constraint that $\sum_{i \geq k} x_{i, \sigma^{k}} \leq B$, where $\sigma^{k}$ denotes the signal whose price point is $k$. 
We write $\widetilde{W}_{(k, B)}(S)$ for the sanitized welfare achieved by the optimum solution with a set of signal price points $S \cup\{k\}$, and the constraint that the probability mass for signal $k$ is at most $B$. Our main lemma is:

LEMMA 5.2. If $S \subseteq T$, then for any $k, B, \epsilon: \widetilde{W}_{(k, B+\epsilon)}(T)-\widetilde{W}_{(k, B)}(T) \leq \widetilde{W}_{(k, B+\epsilon)}(S)-$ $\widetilde{W}_{(k, B)}(S)$.

Lemma 5.2 implies submodularity quite directly, as follows.

Proof of THEOREM 5.1. Let $X$ and $\widehat{X}$ be the optimal signaling schemes with price point sets $S \cup\{k\}$ and $T \cup\{k\}$, respectively. By the monotonicity argument, when constructing $\sigma^{k}$ and $\omega^{k}$, the residual probability for $\sigma^{k}$ is more than that of $\omega^{k}$; therefore, $\widehat{x}_{[k, n], \omega^{k}} \leq x_{[k, n], \sigma^{k}}$ by Lemma 4.2.

Consider gradually increasing $B$ from 0 to $\widehat{x}_{[k, n], \omega^{k}}$ in increments of (varying) $\epsilon$, as outlined above. Subsequently, continue increasing $B$ for $X$ only. By adding up the inequality from Lemma 5.2 for each such step, and noting that the subsequent increases of $B$ for $X$ can only further increase the welfare of $X$, we obtain that $\widetilde{W}(S \cup\{k\})-\widetilde{W}(S) \geq \widetilde{W}(T \cup\{k\})-\widetilde{W}(T)$.

Because the objective function is submodular (and monotone), the greedy algorithm is known [Nemhauser et al. 1978] to give a $(1-1 / e)$-approximation for the problem of maximizing $\widetilde{W}(S)$. Proposition 2.1 then implies that the same greedy algorithm gives a $\frac{M-1}{M} \cdot(1-1 / e)$ approximation for the objective of maximizing the social welfare $W(S)$, proving Theorem 1.3.

The proof of Lemma 5.2 is technically quite involved, and given in the full version. The idea is to first prove it for sufficiently small $\epsilon$, which allows us to couple the executions tightly.

In particular, by comparing the solutions to the linear program (1), we can ensure that any constraint that becomes tight in the solution for set $T$ with bound $B+\epsilon$, but is not tight with bound $B$ (and similarly for $S$ ) would not have become tight for any $\epsilon^{\prime}<\epsilon$. This will localize the changes, and maintain the revenue indifference for the seller. By summing over all such iterations (there will only be finitely many, because $\epsilon$ is chosen so that at least one more constraint becomes tight), we eventually prove the lemma for all $\epsilon$.

\section{REVENUE IN BILATERAL TRADE}

In this section, we prove Theorem 1.4, giving a straightforward dynamic program to compute a signaling scheme maximizing the seller's revenue. Before doing so, we exhibit an equal-revenue distribution for which any signaling scheme with $M$ signals only recovers an $O(M / n)$ fraction of the full-information welfare. Notice again the contrast to the case of welfare maximization, where even one segment is enough to attain an $\Omega(1 / \log n)$ fraction of the full-information welfare.

We define an equal-revenue distribution as follows. Let the valuations be $v_{i}=2^{i}$ for $0 \leq i \leq n$, and the probabilities $p_{i}=\frac{1}{2^{i+1}}$ for $0 \leq i<n$, and $p_{n}=\frac{1}{2^{n}}$. The fullinformation welfare is $\sum_{i=0}^{n} p_{i} v_{i}=\frac{n}{2}+1$. However, for every segment $\boldsymbol{q} \leq \boldsymbol{p}$, no matter what price $v_{i}$ the seller chooses, the revenue cannot be more than $v_{i} \cdot \sum_{j \geq i} p_{j}=1$. Therefore, in the worst case, with $M$ signals, the seller can at best get an $O(\bar{M} / n)$ fraction of the maximum social welfare as his revenue, whereas a fully informed seller would be able to extract the entire full-information welfare, as discussed in the introduction.

Next, we turn our attention to the problem of computing the optimum signaling scheme for the seller's revenue. The key insight enabling a dynamic program is that 
the seller-optimal signaling scheme partitions the buyer types into disjoint intervals, and allocates all probability mass for a given interval to one signal.

Lemma 6.1 (INTERVAl Structure OF SEller-Optimal Signaling Scheme). W.l.o.g., the seller-optimal signaling scheme $X$ has the following form: There are disjoint intervals $I_{1}, I_{2}, \ldots, I_{M}$ of buyer types such that $\bigcup_{\sigma} I_{\sigma}=\{1, \ldots, n\}$, and for each signal $\sigma, x_{i, \sigma}=p_{i}$ for all $i \in I_{\sigma}$ (and $x_{i, \sigma}=0$ for all $i \notin I_{\sigma}$ ).

PROof. Let $k_{1}>k_{2}>\ldots>k_{M}$ be the price points of the signals $\sigma$ under $X$. We will show how to transform $X$ to the claimed form without decreasing the seller's revenue.

First, if $x_{i, \sigma}>0$ for some $\sigma<M, i<k_{\sigma}$, then the buyers of type $i$ will not buy when signal $\sigma$ is sent, contributing nothing to the seller's revenue. Therefore, setting $x_{i, \sigma}=0$ instead does not lower the seller's revenue, and increasing $x_{i, M}$ by the same amount again cannot decrease the seller's revenue. Hence, we may assume that for all signals $\sigma<M$, we have $x_{i, \sigma}>0$ only for $i \geq k_{\sigma}$.

Next, if $x_{i, \sigma}>0$, then $x_{i, \sigma}=p_{i}$. We distinguish two cases: if there is unallocated probability mass of type $i$, then $x_{i, \sigma}$ can simply be raised. If $x_{i, \sigma^{\prime}}>0$ for $\sigma^{\prime}>\sigma$, we can lower $x_{i, \sigma^{\prime}}$ to 0 while raising $x_{i, \sigma}$ by the same amount. Because $\sigma<\sigma^{\prime} \leq M$, we have that $i \geq k_{\sigma}$, so the seller's revenue increases by $x_{i, \sigma^{\prime}} \cdot\left(v_{k_{\sigma}}-v_{k_{\sigma^{\prime}}}\right) \geq 0$.

So far, we have shown that the signals partition the buyer types into sets such that for each buyer type, all of its probability mass goes to its unique designated signal. It remains to show that the partitions are intervals. If not, then there would be two signals $\sigma^{\prime}>\sigma$ and price points $i<i^{\prime}$ such that $x_{i, \sigma}=p_{i}, x_{i^{\prime}, \sigma^{\prime}}=p_{i^{\prime}}$. Then, reallocating the probability mass $x_{i^{\prime}, \sigma^{\prime}}$ to signal $\sigma$ instead increases the seller's revenue by at least $x_{i^{\prime}, \sigma^{\prime}} \cdot\left(v_{k_{\sigma}}-v_{k_{\sigma^{\prime}}}\right) \geq 0$.

The dynamic program for segmentation into intervals is now standard. Let $R(i, m)$ denote the optimal revenue a seller can obtain from buyer types $\{i, i+1, \ldots, n\}$ with $m$ signals, when the lowest price is $v_{i} . R(i, m)$ satisfies the recurrence $R(i, 0)=0$ and $R(i, m)=\max _{i<i^{\prime} \leq n}\left(R\left(i^{\prime}, m-1\right)+v_{i} \cdot \sum_{j=i}^{i^{\prime}-1} p_{j}\right)$. The maximum attainable revenue can be found by exhaustive search of $R(i, M)$ over all $i$.

\section{HARDNESS OF GENERAL PERSUASION}

In this section, we outline the proof of Theorem 1.5 from the introduction. More accurately, we sketch the proof of the following theorem, proving the hardness of maximizing sanitized sender utility within any constant. Details can be found in the full version.

THEOREM 7.1. Unless $P=N P$, for any constant $c>0$, there is no polynomial-time algorithm for the following problem. Given a Bayesian persuasion game $\left(\Omega, \boldsymbol{p}, A, u_{S}, u_{R}\right)$ and cardinality constraint $M$ on the number of signals, construct a signaling scheme $X$ using at most $M$ signals such that the sanitized sender utility $\widetilde{U}(X)$ under $X$ is at least $c \cdot \widetilde{U}\left(X^{*}\right)$, where $X^{*}$ is the signaling scheme maximizing $\widetilde{U}(X)$.

Because the sender utility and sanitized sender utility are within a factor of $\frac{M-1}{M}$ of each other, this implies the same hardness result for the sender utility, proving Theorem 1.5 .

We prove Theorem 7.1 by establishing hardness for a game we call the HYPERGRAPH EDGE GUESSING GAME (HEGG). There is a hypergraph $H=(V, E)$ which is commonly known to the sender and receiver. The state of nature is a hyperedge $e^{*} \in E$, drawn from the uniform distribution.

The receiver has two types of actions available: trying to guess the hyperedge, or "hedging her bets" by guessing a vertex $v \in V$. If she guesses an edge $e$, then she gets 
1 if her guess was correct $\left(e=e^{*}\right)$, and 0 otherwise. If she guesses a vertex $v$, she gets $1 / d_{v}$ (the degree of $v$ ) if $v$ is incident on $e^{*}$, and 0 otherwise.

The sender's utility is determined by the receiver's guess. If the receiver guesses an edge, the sender gets utility 0 , regardless of whether the guess is correct. If the receiver guesses a vertex $v$, the sender has utility $1 / d_{v}$ (the same as the receiver) if $v$ is incident on $e^{*}$, and 0 otherwise.

The approximation hardness of HEGG is proved by a reduction from the gap version of INDEPENDENT SET [Zuckerman 2006]. When establishing an approximation hardness of $1 / r$, the reduction on a graph $G$ runs in time $\Theta\left(n^{r+1}\right)$, building a hypergraph of all $(r+1)$-cliques of $G$. The proof relies on Ramsey's Theorem.

\section{CONCLUSION}

Our work raises several natural questions for future work. First, while we provide a constant-factor approximation algorithm and a QPTAS for social welfare in the pricing game, we did not actually establish NP-hardness. Is there a polynomial-time algorithm for maximizing social welfare subject to limited communication? Could at least a PTAS or an FPTAS be obtained? For the more general persuasion problem with limited communication, we establish that no approximation of social welfare to within any constant is possible. Can this result be strengthened to logarithmic or polynomial hardness?

In the present submission, we are focusing on maximizing seller revenue and social welfare. Bergemann et al. [2015] also consider maximizing buyer's utility. It is not hard to see that given the price points for each signal, the buyer's utility can be maximized by a linear program very similar to (1). However, it is not clear that the overall objective function is still submodular, or whether a similar greedy algorithm to the one from Section 4 optimally solves the corresponding LP.

Beyond revenue and social or buyer welfare, one could consider other objectives for the principal. While for full generality of the persuasion problem, our results preclude constant-factor approximation guarantees, it would be of interest to identify other natural classes in which limits on communication have mild consequences, and in which good signaling schemes with limited communication can be designed efficiently.

Acknowledgments. We would like to thank Alex Eager for useful conversations and anonymous reviewers for useful feedback.

\section{REFERENCES}

George A. Akerlof. 1970. The Market for "Lemons": Quality Uncertainty and the Market Mechanism. The Quarterly Journal of Economics 84, 3 (August 1970), 488-500.

Ricardo Alonso and Odilon Camara. 2014. Persuading Voters. (2014). Working Paper.

Dirk Bergemann, Benjamin Brooks, and Stephen Morris. 2015. The Limits of Price Discrimination. American Economic Review 105, 3 (2015), 921-957.

Liad Blumrosen and Michal Feldman. 2006. Implementation with a bounded action space. In Proc. 8th ACM Conf. on Electronic Commerce. ACM, 62-71.

Liad Blumrosen, Noam Nisan, and Ilya Segal. 2007. Auctions with severely bounded communication. Journal of Artificial Intelligence Research 28 (2007), 233-266.

Peter Bro Miltersen and Or Sheffet. 2012. Send mixed signals: Earn more, work less. In Proc. 14th ACM Conf. on Electronic Commerce. 234-247.

Isabelle Brocas and Juan D. Carrillo. 2007. Influence through ignorance. The RAND Journal of Economics 38, 4 (2007), 931-947.

Yu Cheng, Ho Yee Cheung, Shaddin Dughmi, Ehsan Emamjomeh-Zadeh, Li Han, and Shang-Hua Teng. 2015. Mixture Selection, Mechanism Design, and Signaling. In Proc. 56th IEEE Symp. on Foundations of Computer Science. 1426-1445. 
Shaddin Dughmi. 2014. On the Hardness of Signaling. In Proc. 55th IEEE Symp. on Foundations of Computer Science. 354-363.

Shaddin Dughmi, Nicole Immorlica, Ryan O'Donnell, and Li-Yang Tan. 2015. Algorithmic Signaling of Features in Auction Design. In Algorithmic Game Theory - 8th International Symposium, SAGT 2015, Saarbrücken, Germany, September 28-30, 2015, Proceedings. 150-162.

Shaddin Dughmi, Nicole Immorlica, and Aaron Roth. 2014. Constrained Signaling in Auction Design. In Proc. 25th ACM-SIAM Symp. on Discrete Algorithms. 1341-1357.

Shaddin Dughmi and Haifeng Xu. 2016. Algorithmic Bayesian Persuasion. In Proc. 47th ACM Symp. on Theory of Computing.

Yuval Emek, Michal Feldman, Iftah Gamzu, Renato Paes Leme, and Moshe Tennenholtz. 2012. Signaling Schemes for Revenue Maximization. In Proc. 14th ACM Conf. on Electronic Commerce. 514-531.

Wolfgang Gick and Thilo Pausch. 2012. Persuasion by stress testing: Optimal disclosure of supervisory information in the banking sector. (2012). Discussion Paper 32/2012, Deutsche Bundesbank.

Itay Goldstein and Yaron Leitner. 2013. Stress tests and information disclosure. (2013). FRB of Philadelphia Working Paper.

Mingyu Guo and Argyrios Deligkas. 2013. Revenue Maximization via Hiding Item Attributes. In Proc. 28th Intl. Joint Conf. on Artificial Intelligence.

Emir Kamenica and Matthew Gentzkow. 2011. Bayesian Persuasion. American Economic Review 101, 6 (2011), 2590-2615. DOI : http://dx.doi.org/10.1257/aer.101.6.2590

Anton Kolotilin. 2015. Experimental design to persuade. Games and Economic Behavior 90 (2015), 215-226.

Ilan Kremer, Yishay Mansour, and Motty Perry. 2014. Implementing the "Wisdom of the Crowd”. Journal of Political Economy 122, 5 (2014), 988-1012.

Jonathan Levin and Paul Milgrom. 2010. Online advertising: Heterogeneity and conflation in market design. American Economic Review 100, 2 (2010), 603-607.

Yishay Mansour, Aleksandrs Slivkins, and Vasilis Syrgkanis. 2015. Bayesian Incentive-Compatible Bandit Exploration. In Proc. 17th ACM Conf. on Economics and Computation. 565-582.

Andreu Mas-Collel, Michael D. Whinston, and Jerry R. Green. 1995. Microeconomic Theory. Oxford University Press.

R. Myerson. 1981. Optimal Auction Design. Mathematics of Operations Research 6 (1981), 58-73.

George L. Nemhauser, Laurence A. Wolsey, and Marshall L. Fisher. 1978. An analysis of the approximations for maximizing submodular set functions. Mathematical Programming 14 (1978), 265-294.

A. Pigou. 1920. The Economics of Welfare. Macmillan.

Zinovi Rabinovich, Albert Xin Jiang, Manish Jain, and Haifeng Xu. 2015. Information Disclosure as a Means to Security. In Proc. 14thIntl. Conf. on Autonomous Agents and Multiagent Systems. 645-653.

Anne-Katrin Roesler and Balázs Szentes. 2016. Buyer-Optimal Learning and Monopoly Pricing. Technical Report. Mimeo, London School of Economics.

Haifeng Xu, Zinovi Rabinovich, Shaddin Dughmi, and Milind Tambe. 2015. Exploring Information Asymmetry in Two-Stage Security Games. In Proc. 30th AAAI Conf. on Artificial Intelligence. 1057-1063.

David Zuckerman. 2006. Linear degree extractors and the inapproximability of max clique and chromatic number. In Proc. 37th ACM Symp. on Theory of Computing. ACM, 681-690. 